\title{
Differential Diagnosis and Treatment of Comorbid PTSD and TBI in Combat Veterans
}

\author{
Jennifer Clees \\ College of Liberal Arts and Sciences, University of Florida
}

Faculty mentor: Daiqing Liao, Department of Anatomy and Cell Biology, College of Medicine

\begin{abstract}
The purpose of this paper was to examine what is currently known about diagnosing and treating comorbid PTSD and TBI in combat veterans, beginning with a focus on differential diagnosis of the two disorders in order to elucidate any obfuscation by overlaps in symptomology, and concluding with possible treatment plans, as informed by the diagnostic process. Differential diagnosis was evaluated via self-reported and clinician-administered questionnaires, oculomotor testing, and neuroimaging. Selfreporting diagnostic tools are quick and easy to screen for PTSD and TBI, but these methods may overreport PTSD and under-report TBI. Clinician-administered questionnaires are longer and require a trained professional, but accuracy is gained. The oculomotor testing and SPECT scanning appease a more impartial evidence-based approach, and further experimentation could reinforce the usefulness of both to military, VA, and civilian medical facilities. Meanwhile, the discussion of treatment options acknowledged the necessity of adjustments to traditional treatment paradigms when a comorbid diagnosis is involved. Psychotherapy is generally the number one recommended treatment for PTSD and TBI, due to its efficacy compared to pharmaceuticals, and further studies elucidating the efficacy of varieties of psychotherapy treatment may benefit individuals with this comorbid diagnosis.
\end{abstract}

Keywords: traumatic brain injury, differential diagnosis, PTSD

\section{Introduction}

Among the nearly 2 million United States Armed Forces combat veterans returning from Operation Enduring Freedom (OEF) (2001-2014) and Operation Iraqi Freedom (OIF) (20032010), later renamed Operation New Dawn (2010-2011), it is estimated that $20 \%$ experience a traumatic brain injury (TBI) ${ }^{1,2}, 10-20 \%$ leave with post-traumatic stress disorder (PTSD) $)^{2,3,4,5}$, and comorbidity may occur in $30-40 \%$ of these individuals ${ }^{2,4,5}$. However, the variety of diagnostic tools utilized produces modulated prevalence estimates- some lower, others higher ${ }^{2}$. As little as ten year ago, researchers studied whether the comorbidity of PTSD and TBI was folklore or fact, with some arguing the TBI would prevent PTSD from occurring, as the traumatic event may physiologically produce gaps in memory ${ }^{6}$. The purpose of this paper is to examine what is currently known about diagnosing and treating comorbid PTSD and TBI in combat veterans, beginning with a focus on differential diagnosis of the two disorders in order to 
elucidate any obfuscation by overlaps in symptomology, and concluding with possible treatment plans, as informed by the diagnostic process.

\section{Background}

Combat veterans with PTSD may present with re-experiencing, hyperarousal, avoidance, and emotional numbing as a result of traumatic experiences including being in life-or-death situations, seeing friends die, and engaging in combat. Clinicians use the Diagnostic and Statistical Manual of Mental Disorders (DSM-IV-TR from 2000-2013, now the DSM-5) to establish whether an individual meets the diagnostic criteria for PTSD. This criteria is summarized as follows: (1) exposure to a traumatic event, (2) intrusive recollection of the event through unwanted upsetting memories, nightmares, and other ways, (3) avoidance of traumarelated stimuli after the trauma, (4) negative thoughts or feelings that began or worsened after the trauma, such as negative affect, feeling isolated, or decreased interest in activities, (5) traumarelated arousal and reactivity that began or worsened after the trauma, such as irritability, hypervigilance, difficulty sleeping, or difficulty concentrating, (6) symptom duration is greater than one month, and (7) symptoms create distress or functional impairment (e.g., social, occupational $)^{7}$. Although PTSD is not unique to the twenty-first century, with the frequent use of improvised explosive devices (IEDs) in modern warfare, head and neck injuries, particularly blast-related TBIs occur more frequently nowadays than documented in any prior wars ${ }^{1,3}$. Another contributor may be improved body armor which prevents cardiothoracic blast injuries that would have killed soldiers in the past, but does not prevent TBIs, also known as concussions $^{3}$. Blunt head trauma from accidental aircraft and motor vehicle crashes, though less novel, also adds to combat TBI rates. Individuals with TBI may experience an alteration/loss of consciousness, amnesia, or neurological deficits ${ }^{3}$. To be diagnosed with TBI after head trauma, an individual must have a new onset or a worsening of at least one of the following symptoms: (1) any period of loss of or a decreased level of consciousness (LOC), (2) any loss of memory for events immediately before or after the injury (posttraumatic amnesia [PTA]), (3) any alteration in mental state at the time of the injury (confusion, disorientation, slowed thinking, etc.), (4) neurological deficits (weakness, loss of balance, change in vision, praxis, paresis/plegia, sensory loss, aphasia, etc.) that may or may not be transient, and/or intracranial lesion ${ }^{7}$. A study of 1648 military personnel in the Marines and Navy found that a TBI during the most recent deployment 
was the strongest predictor of post-deployment PTSD ${ }^{1}$. Additionally, overlapping symptoms of PTSD and TBI may include poor concentration, memory difficulties, anhedonia, or lack of interest or pleasure in doing things, social isolation, sleep difficulties, depression, anxiety, pain, and irritability ${ }^{5,7}$. Along these lines, PTSD and reduced concentration may affect memory, but TBI could also directly damage brain tissues and impact memory consolidation ${ }^{3}$. Whether the combat veteran has PTSD, a TBI, or both, the impact of a traumatic event can persist and follow them even after they have left the military, affecting their daily lives and families, and these overlaps in symptomology contribute to difficulties in ascribing a symptom to a specific diagnosis if both are present and in differential diagnosis, particularly when self-reported assessments are used ${ }^{5,7}$.

\section{Diagnosis}

\section{Questionnaires (Self-Reported and Clinician-Administered)}

A Post-Deployment Health Assessment (PDHA) is given to all service members returning from deployment, and a Post-Deployment Health Reassessment (PDHRA) is generally given 3 to 6 months after deployment ${ }^{9}$. Responding yes to two of the Primary Care 4-item Post-Traumatic Stress Disorder screen (PC-PTSD) questions on the PDHA or PDHRA is considered screening positive for PTSD ${ }^{9}$. While these assessments inquire about combat exposure and current symptoms of PTSD, it was not until 2008 that the PDHA and PDHRA included questions indicating a risk of TBI exposure ${ }^{9}$. A latent class analysis study using data from 12,581 veterans found the rate of TBI underdiagnosis in the PDHA/PDHRA to be $4.63 \%{ }^{9}$.

Upon entering the Veterans Administration (VA) hospital system, as of 2007, military personnel are screened again for TBI, but in this instance a clinician administers the 4-item screen, and positive questionnaires result in recommendation to polytrauma clinics ${ }^{9,10}$. Positive screening results only indicate those at risk for TBI but have often been confused for medical record documentation of deployment $\mathrm{TBI}^{10}$. In addition, the VA TBI screen is designed to be more sensitive than specific, meaning true positives are identified at a higher rate than true negatives are not identified ${ }^{10}$. When a study of 179 veterans' VA TBI screen results were compared to the Boston Assessment of Traumatic Brain Injury-Lifetime (BAT-L) clinician interview, 2 veterans with blast injuries and one with blunt head trauma from a motor vehicle collision while on combat patrol screened negative with the VA questions, but screened positive 
with the BAT-L ${ }^{10}$. The incorrect belief that loss of consciousness must result for an injury to be a TBI, or the presence of more pressing, severe bodily injuries may lead to underdiagnosis of $\mathrm{TBI}^{3}$.

On the other hand, the self-reported PTSD Checklist (PCL) resulted in 20\% higher rates of PTSD than the Clinician-Administered PTSD Scale (CAPS) ${ }^{3}$. Both the PCL and CAPS surveys are based on the Diagnostic and Statistical Manual of Mental Disorders (DSM-IV-TR) and have been tested for reliability, but the CAPS is longer and delineates between severity and frequency of symptoms ${ }^{2}$. One study found a history of TBI was associated with higher self- and clinicianrated PTSD symptoms, using the PCL and CAPS, respectively ${ }^{11}$. In a separate study, researchers had to control for post-concussive symptoms in order for veterans with blast exposure to have similar PCL scores to those without $\mathrm{TBI}^{12}$.

\section{Oculomotor Testing}

While TBI and PTSD symptoms can overlap and cloud the path to diagnosis, oculomotor function, or eye movement tests, can be used as a noninvasive, auxiliary tool for TBI diagnosis and are not known to be confounded by psychiatric comorbidities or antidepressant medication ${ }^{3}$. Changes in visual function are associated with TBI but have not been documented secondary to PTSD $^{7}$. In one study, patients with comorbid PTSD and TBI self-reported more visual symptoms and higher levels of light sensitivity and reading problems, but the high rates of oculomotor function deficits, such as $71 \%$ saccadic dysfunction and $67 \%$ accommodative insufficiency, of patients with TBI did not differ significantly between patients with or without comorbid PTSD $^{7}$. Oculomotor testing evaluates gaze stabilization, vestibule-ocular reflex, optokinetic reflex, tracking/ smooth pursuit, saccades, vergence, and pupillary light reflex ${ }^{3,7}$. At least one abnormal finding occurred in $30 \%$ of veterans exposed to blasts, and the testing may allow the clinician to determine the damaged brain area in question such as the brain stem or cerebellum ${ }^{13}$. The study also claims that clinicians are easily able to identify feigned or exaggerated results ${ }^{3}$. While individuals may lack awareness of their cognitive deficits, oculomotor testing allows for more objective testing of vestibular, ocular, and neurological systems ${ }^{3}$.

\section{Neuroimaging}

The default mode network (DMN) consists of various areas in the brain that are active 'by default' when an individual is not participating in a task and can act as a biomarker of disease ${ }^{5}$. 
A dose of technetium-99m exametazime is administered intravenously at rest and while performing a concentration task, with scans being taken 30 minutes after injection ${ }^{5}$. Brain single photon emission computed tomography (SPECT) images of the DMN indicate increased blood flow in PTSD, decreased blood flow in TBI, and intermediate blood flow in PTSD/TBI, but this intermediate perfusion pattern is still noticeably different from healthy controls ${ }^{5}$. The SPECT imaging has the ability to differentiate between veterans, previously diagnosed without neuroimaging, with PTSD from those with TBI with 87-94\% accuracy, TBI from PTSD/TBI with 79-83\% accuracy, and PTSD from PTSD/TBI with 85-92\% accuracy ${ }^{5}$. If replicate studies demonstrate similar reliability in differential diagnosis, then SPECT imaging holds the promise to be clinically useful not only in diagnosis, but also in showing patients pictures of the physiological processes occurring in the brain. These pictures could perhaps help reduce stigma, self-blame, and treatment attrition ${ }^{5}$.

\section{Treatment}

As a clinician, a comorbid PTSD/ TBI diagnosis informs the treatment plan in that a TBI may necessitate certain adjustments to a traditional PTSD treatment plan. For example, if pharmaceuticals are given, paroxetine, fluoxetine, and tricyclic antidepressants will worsen the symptoms of the TBI, but serotonin-reuptake inhibitors (SSRIs) and serotonin-norepinephrinereuptake inhibitors (SNRIs) are safe ${ }^{3,8}$. Psychotherapies such as virtual reality exposure therapy, prolonged exposure (PE), cognitive processing therapy (CPT), or CPT-cognitive only (CPT-C) may be used $2,3,4,11,14$. PTSD/TBI patients commonly report lack of memory of the traumatic event, but remain open to discussing current symptoms ${ }^{2}$. Therefore, more time and emotional processing may be necessary to address such memory gaps ${ }^{2}$. Increasing the length of sessions or number of sessions to spread out and repeat exercises or content may allow treatment to be more effective and personalized to the veteran ${ }^{2}$. Flexibility to accommodate endocrine, sleep, and substance abuse disorders may also impact the treatment plan ${ }^{3}$.

\section{Conclusion}

TBI and PTSD have become the 'signature wounds' of combat veterans returning to the United States from deployment, and sustained military involvement in the Middle East with concerns of ISIS suggest thousands more service members will be in harm's way. Troops will 
still experience traumas, and it is doubtful that helmet armor technology can protect all soldiers from absolutely all head injuries. While research into the comorbidity of TBI and PTSD has exploded over the past decade, troop education on the signs and symptoms of TBI, even without loss of consciousness, and a protocol for them to report suspected TBI events, may contribute to awareness and openness towards diagnosis and treatment.

Self-reporting diagnostic tools are quick and easy to screen for PTSD and TBI, but these methods may over-report PTSD and under-report TBI, with shared symptoms muddling the waters. Clinician-administered questionnaires are longer and require a trained professional, but accuracy is gained. The wealth of surveys may leave the military and VA questioning which ones are best, and besides the PCL vs. CAPS, and VA TBI screen vs. BAT-L, no large body of research currently exists comparing inter-survey reliability. Both diagnostic methods lack a more objective approach to ruling out one disorder or the other when not comorbid because these surveys rely upon descriptions of symptoms including symptoms that overlap in PTSD and TBI. The oculomotor testing and SPECT scanning appease a more impartial evidence-based approach, and further experimentation could reinforce the usefulness of both to military, VA, and civilian medical facilities.

Psychotherapy is generally the number one recommended treatment for PTSD and TBI due to its efficacy compared to pharmaceuticals. A study presently in randomized clinical trials is testing the effect of SMART-CPT, a therapy joining the treatment of emotional symptoms by CPT to treatment of cognitive symptoms by CogSMART ${ }^{4}$. Researchers hypothesize that this therapy tailored to patients with PTSD and TBI will be more effective than CPT alone ${ }^{4}$. In terms of pharmaceutical treatments, some TBI contraindications for PTSD, sleep, and depression drugs exist in literature, but many more drugs call for supplementary research as there is no advice one way or another. Attrition rate, cost, time commitment, and travel time investigations would contribute to clinician knowledge of treatment feasibility.

Due to the challenging diagnosis and management of care for a combat veteran with PTSD and TBI, clinicians should be prepared to implement an individualized treatment plan grounded in clinical practice guidelines, led by an outpatient case manager, and carried out by an interdisciplinary team. New literature may supplement the diagnostic tools and treatment plan chosen by investigating which questions or questionnaires best inform the clinician of the combat veteran's physical and mental status following exposure to trauma, as verified by current 
methods such as oculomotor testing or SPECT scanning, or by future discoveries in randomized clinical trials. PTSD and TBI can drastically change a combat veteran's life, but proper diagnosis and care can lessen or eliminate the burden of symptoms.

\section{References}

1. Yurgil KA, Barkauskas DA, Vasterling JJ, et al. Association between traumatic brain injury and risk of posttraumatic stress disorder in active-duty marines. JAMA Psychiatry. 2014;71(2):149-157. doi: 10.1001/jamapsychiatry.2013.3080.

2. Strom TQ, Wolf GK, Crawford E, Blahnik M, Kretzmer T. Implementing Prolonged Exposure for Veterans with Comorbid PTSD and Traumatic Brain Injury: Two Case Studies. Cogn Behav Pract. 2016;23:148-161. doi: 10.1016/j.cbpra.2015.03.003.

3. Capehart BP, Bass D. Review: Managing posttraumatic stress disorder in combat veterans with comorbid traumatic brain injury. J Rehabil Res Dev. 2012;49(5):789-812. doi: 10.1682/JRRD.2011.10.0185.

4. Jak A, Aupperle R, Rodgers C, et al. Evaluation of a hybrid treatment for veterans with comorbid traumatic brain injury and posttraumatic stress disorder: Study protocol for a randomized controlled trial. Contemp Clin Trials. 2015;45(Part B):210-216. doi: 10.1016/j.cct.2015.10.009.

5. Raji CA, Willeumier K, Taylor D, et al. Functional neuroimaging with default mode network regions distinguishes PTSD from TBI in a military veteran population. Brain Imaging and Behavior. 2015;9:527-534. doi:10.1007/s11682-015-9385-5.

6. King NS. PTSD and traumatic brain injury: Folklore and fact? Brain Injury. 2008;22(1):1-5. doi: 10.1080/02699050701829696.

7. Goodrich GL, Martinsen GL, Flyg HM, Kirby J, Garvert DW, Tyler CW. Visual function, traumatic brain injury, and posttraumatic stress disorder. J Rehabil Res Dev. 2014;51(4):547-558. doi: 10.1682/JRRD.2013.02.0049.

8. Huggins JM, Brown JN, Capehart BP, Townsend ML, Legge J, Melnyk SD. Medication adherence in combat veterans with traumatic brain injury. Am J Health-Syst Pharm. 2011;68:254-258. doi: 10.2146/ajhp090629.

9. Aralis H, Macera C, Rauh M, MacGregor A. Traumatic Brain Injury and PTSD Screening Efforts Evaluated Using Latent Class Analysis. Rehabil Psychol. 2014;59:68-78. doi: 10.1037/a0035263.

10. Fortier C, Amick M, Kenna A, Milberg W, McGlinchey R. Correspondence of the Boston Assessment of Traumatic Brain Injury-Lifetime (BAT-L) Clinical Interview and the VA TBI Screen. J Head Trauma Rehab. 2015;2013;30:E1-E7. doi: 10.1097/HTR.0000000000000008

11. Davis JJ, Walter KH, Chard KM, Parkinson RB, Houston WS. Treatment adherence in cognitive processing therapy for combat-related PTSD with history of mild TBI. Rehabil Psychol. 2013;58(1):36-42. doi: 10.1037/a0031525. 
12. Levin HS, Wilde E, Troyanskaya M, et al. Diffusion tensor imaging of mild to moderate blast-related traumatic brain injury and its sequalae. J Neurotrauma. 2010;27(4):683-94. doi: 10.1089/neu.2009.1073.

13. Capehart BP, Mehlenbacher A, Smith-Hammond C, Bass D, Burke J. Blast exposure and oculomotor function. American Neurologic Association annual scientific meeting; 2011 Sep; San Diego, CA.

14. Walter KH, Dickstein BD, Barnes SM, Chard KM. Comparing effectiveness of CPT to CPT-C among U.S. veterans in an interdisciplinary residential PTSD/TBI treatment program. J Trauma Stress. 2014;27(4):438-445. doi: 10.1002/jts.21934. 